\title{
On the entropy method and exponential convergence to equilibrium for a recombination-drift-diffusion system with self-consistent potential
}

\author{
Klemens Fellner ${ }^{\mathrm{a}, *}$, Michael Kniely ${ }^{\mathrm{a}}$ \\ ${ }^{a}$ Karl-Franzens-Universität Graz, Heinrichstraße 36, 8010 Graz
}

\begin{abstract}
We consider a Shockley-Read-Hall recombination-drift-diffusion model coupled to Poisson's equation and subject to boundary conditions, which imply conservation of the total charge. As main result, we derive an explicit functional inequality between relative entropy and entropy production rate, which implies exponential convergence to equilibrium with explicit constant and rate. We report that the key entropy-entropy production inequality ought rather not to be formulated on the states space of the parabolic-elliptic system, but on the reduced states space of the associated nonlocal drift-diffusion problem, where the Poisson equation is replaced by the corresponding Green function.
\end{abstract}

Keywords: Semiconductor model, Shockley-Read-Hall recombination, drift-diffusion systems, self-consistent potential, entropy method, convergence to equilibrium

2010 MSC: 35B40, 82D37, 35K57

\section{Introduction}

This paper investigates the large-time-behaviour of the following recombination-drift-diffusion-Poisson system on a bounded Lipschitzian domain $\Omega \subset \mathbb{R}^{m}$ :

$$
\begin{cases}\partial_{t} n=\nabla \cdot J_{n}(n, \psi)-R(n, p), & J_{n}:=\mu_{n}\left(\nabla n+n \nabla\left(\psi+V_{n}\right)\right), \\ \partial_{t} p=\nabla \cdot J_{p}(p, \psi)-R(n, p), & J_{p}:=\mu_{p}\left(\nabla p+p \nabla\left(-\psi+V_{p}\right)\right), \\ -\varepsilon \Delta \psi=n-p-C, & \end{cases}
$$

where $n, p$ and $\psi$ represent the concentrations of electrons and holes as well as the self-consistent electric potential. The recombination terms are of Shockley-Read-Hall form, i.e.

$$
R:=F(n, p, x)\left(n p-e^{-V_{n}-V_{p}}\right), \quad 0<C_{\mathrm{F}} \leq F(n, p, x),
$$

and $V_{n}, V_{p} \in H^{1}(\Omega) \cap L^{\infty}(\Omega)$ are external potentials. The strictly positive functions $\mu_{n}$ and $\mu_{p}$ with $\mu_{n}, \mu_{p} \geq \mu>0$ a.e. in $\Omega$ denote scaled mobilities of electrons and holes under the assumption that the Einstein relations hold true, see e.g. [MRS90]. In addition, $C \in L^{\infty}(\Omega)$ describes the internal doping profile and $\varepsilon>0$ is the permittivity constant. Note that the drift-diffusion fluxes $J_{n}$ and $J_{p}$ can also be written in terms of the quasi-Fermi potentials $\Phi_{n}$ and $\Phi_{p}$, i.e.

$$
J_{n}=\mu_{n} n \nabla \Phi_{n}, \quad \Phi_{n}:=\psi+V_{n}+\ln n, \quad J_{p}=\mu_{p} p \nabla \Phi_{p}, \quad \Phi_{p}:=-\psi+V_{p}+\ln p .
$$

The main aim of the paper is to prove exponential convergence to an equilibrium state $\left(n_{\infty}, p_{\infty}, \psi_{\infty}\right)$ of (suitable) solutions to (12-2) via the so-called entropy method. In our context, the entropy method aims to quantify the decay of the non-negative relative entropy functional

$$
E(n, p, \psi)=\int_{\Omega}\left(n \ln \frac{n}{n_{\infty}}-\left(n-n_{\infty}\right)+p \ln \frac{p}{p_{\infty}}-\left(p-p_{\infty}\right)\right) d x+\frac{\varepsilon}{2} \int_{\Omega}\left|\nabla\left(\psi-\psi_{\infty}\right)\right|^{2} d x \geq 0
$$

\footnotetext{
${ }^{*}$ Corresponding author
}

Email addresses: klemens.fellner@uni-graz.at (Klemens Fellner), michael.kniely@uni-graz.at (Michael Kniely) 
according to the non-negative entropy production functional $P:=-\frac{d}{d t} E$, i.e.

$$
P(n, p, \psi)=\int_{\Omega}\left(\mu_{n} n\left|\nabla \Phi_{n}\right|^{2}+\mu_{p} p\left|\nabla \Phi_{p}\right|^{2}+F(n, p, x)\left(n p-e^{-V_{n}-V_{p}}\right) \ln \left(\frac{n p}{e^{-V_{n}-V_{p}}}\right)\right) d x \geq 0,
$$

in terms of a functional inequality, a so-called entropy-entropy production inequality of the form,

$$
P(n, p, \psi) \geq C_{\mathrm{EEP}} E(n, p, \psi),
$$

for a constant $C_{\mathrm{EEP}}>0$. Note that (5) applied to (sufficiently regular) solutions of system (1)-(2) implies exponential (with rate $C_{\mathrm{EEP}}$ ) convergence to the equilibrium $\left(n_{\infty}, p_{\infty}, \psi_{\infty}\right)$ in the relative entropy $E$ via a Gronwall argument.

The entropy method, conceptionally reaching back to pioneering ideas of Boltzmann in the field of kinetic theory, constitutes a fully nonlinear approach in studying the large-time-behaviour of entropy producing PDEs, which has the advantage of being quite robust as it is based on functional inequalities (which can be reused in related problems) rather than PDE-specific a-priori estimates on solutions. Related previous works using various (non-explicit and explicit) variants of the entropy method are e.g. [Jün95, GGH96, GG96, GH97] for semiconductor models with selfconsistent potential. More generally for chemical reaction-diffusion systems, we refer to the pivotal works of Gröger [Grö83, Grö92] and remark that for systems with conservation laws, explicit proofs of the entropy-entropy production inequality (5) are technically challenging and were first obtained in [DF06, DF08, GZ10, DF14, MHM15, HHMM] for special systems and recently in [FT17, DFT17] for general detailed/complex balanced reaction-diffusion systems.

In the current paper, we study the large-time-behaviour of system (11)-(2) subject to no-flux boundary conditions for $J_{n}$ and $J_{p}$ and homogeneous Neumann boundary conditions for the potential $\psi$ :

$$
\hat{n} \cdot J_{n}=\hat{n} \cdot J_{p}=0 \quad \text { on } \partial \Omega \quad \text { and } \quad \hat{n} \cdot \nabla \psi=0 \quad \text { on } \partial \Omega,
$$

where $\hat{n}$ denotes the outer unit normal vector on the boundary $\partial \Omega$ and we shall assume (w.l.o.g. by rescaling the space variable) that the volume of $\Omega$ is normalised, i.e. $|\Omega|=1$. As a consequence of the no-flux boundary conditions and the equations for $n$ and $p$, we observe that the total charge is conserved:

$$
\overline{n(t)}-\overline{p(t)}=\overline{n_{I}}-\overline{p_{I}}=\bar{C} \in \mathbb{R} \quad \text { for all } \quad t \in \mathbb{R}_{+},
$$

where we denote the spatial average of a function $f$ as $\bar{f}:=\int_{\Omega} f(x) d x$ (recall $|\Omega|=1$ ) and the second equality is a consequence of supposing initial concentrations $n_{I}$ and $p_{I}$, which satisfy charge neutrality, i.e.

$$
\overline{n_{I}}-\overline{p_{I}}=\bar{C} .
$$

Note that the conservation of charge in (7) implies that the system preserves charge neutrality for all times $t \geq 0$. Moreover, charge neutrality constitutes the necessary and sufficient compatibility condition for solving the inhomogeneous Poisson equation in (1) subject to the homogeneous Neumann boundary data (6):

Remark 1.1. Consider the inhomogeneous Poisson equation subject to homogeneous Neumann boundary conditions, i.e.

$$
-\varepsilon \Delta \psi=f \in L^{2} \quad \text { in } \Omega, \quad \hat{n} \cdot \nabla \psi=0 \quad \text { on } \partial \Omega .
$$

Then, there exists a weak solution $\psi \in H^{1}(\Omega)$ if and only if $\bar{f}=0$ (compatibility condition with homogeneous Neumann boundary conditions), which corresponds to $\bar{n}-\bar{p}=\bar{C}$ for the Poisson equation in (11). Moreover, since $\psi$ is determined only up to an additive constant, we choose the normalisation $\bar{\psi}=0$ to obtain a unique solution $\psi$.

The charge conservation law (7) and the charge neutrality (8) are also essential to determine the unique equilibrium state of system (17)-(2) and (6). Based on e.g. [MRS90], [WMZ08] and [Trö09], one can prove the following result.

Proposition 1.2 (Equilibrium States). The stationary state system

$$
\left\{\begin{array}{llr}
\nabla \cdot J_{n}(n, \psi)-R(n, p)=0, & \hat{n} \cdot J_{n}=0 & \text { on } \partial \Omega, \\
\nabla \cdot J_{p}(p, \psi)-R(n, p)=0, & \hat{n} \cdot J_{p}=0 & \text { on } \partial \Omega, \\
-\varepsilon \Delta \psi=n-p-C, & \hat{n} \cdot \nabla \psi=0 & \text { on } \partial \Omega
\end{array}\right.
$$


has a unique equilibrium solution $\left(n_{\infty}, p_{\infty}, \psi_{\infty}\right)$ with $\overline{\psi_{\infty}}=0$, where $\psi_{\infty}$ is continuous and bounded and

$$
n_{\infty}=C_{n} e^{-\psi_{\infty}-V_{n}} \quad \text { and } \quad p_{\infty}=C_{p} e^{\psi_{\infty}-V_{p}}
$$

for two constants $C_{n}, C_{p}>0$ with $C_{n} C_{p}=1$.

Thus, the uniqueness of the equilibrium state is a consequence of the charge conservation law $\overline{n_{\infty}}-\overline{p_{\infty}}=\bar{C}$, i.e.

$$
C_{n} \overline{e^{-\psi_{\infty}-V_{n}}}-C_{p} \overline{e^{\psi_{\infty}-V_{p}}}=\bar{C}
$$

The key step of the entropy method is to establish an entropy-entropy production inequality of the form (5) on a suitable set of admissible functions, for which holds that all admissible states with zero entropy production $P(n, p, \psi)=0$ equal the states with zero relative entropy $E(n, p, \psi)=0$, which is exactly the equilibrium state $\left(n_{\infty}, p_{\infty}, \psi_{\infty}\right)$ and which is determined uniquely by the charge conservation law (11). For the entropy production functional, however, all states of the form

$$
N_{a, \psi}:=a e^{-\psi-V_{n}}, \quad P_{a, \psi}:=a^{-1} e^{\psi-V_{p}}, \quad \text { for any constant } a>0
$$

yield $P\left(N_{a, \psi}, P_{a, \psi}, \psi\right)=0$. Due to the family $\left(N_{a, \psi}, P_{a, \psi}, \psi\right)$, an entropy-entropy production inequality of the form (5) can only hold by taking into account that the potential $\psi$ has to satisfy the inhomogeneous Poisson equation (9) with $f=N_{a, \psi}-P_{a, \psi}-C$ subject to the compatibility condition $\overline{N_{a, \psi}}-\overline{P_{a, \psi}}=\bar{C}$, which corresponds to the charge conservation law (11) and equally implies $\left(N_{a, \psi}, P_{a, \psi}, \psi\right)=\left(n_{\infty}, p_{\infty}, \psi_{\infty}\right)$.

The first interesting result of this paper is the observation, that while the charge conservation law (7) is necessary for an entropy-entropy production inequality of the form (5) as stated in the following Theorem 1.3 , the actual proof does not explicitly use it. In fact, the proof adapts successfully arguments from [GG96], where the uniqueness of the equilibrium is a consequence of thermodynamically consistent mixed Dirichlet-Neumann boundary conditions instead of the charge conservation law (7), which does not hold in the model of [GG96]. Hence, one would believe that the approach of [GG96] cannot really work for the here considered system.

The reason why this point of view is mistaken and why the approach of [GG96] can be successfully adapted lies in the fact that the solvability condition of the elliptic problem (9) is equivalent to the charge conservation law (7). It is as surprising as interesting that the presented proof succeeds in showing Theorem 1.3 by using the charge conservation law (7) only implicitly via the very definition of the considered potential $\psi$ including the normalisation $\bar{\psi}=0$.

Theorem 1.3 (Entropy-Entropy Production Inequality). Consider $\psi_{\infty} \in L^{\infty}(\Omega)$ and define

$$
K_{\infty}:=\left\|\psi_{\infty}\right\|_{L^{\infty}(\Omega)} \quad \text { and } \quad V_{\infty}:=\max \left\{\left\|V_{n}\right\|_{L^{\infty}(\Omega)},\left\|V_{p}\right\|_{L^{\infty}(\Omega)}\right\} .
$$

Then, for all non-negative $n, p \in L^{2}(\Omega)$, for which $\psi=\psi(n, p) \in H^{1}(\Omega)$ is the unique solution of (9) with $f=n-p-C$ and $\bar{\psi}=0$, there exists an explicitly computable constant $C_{\mathrm{EEP}}>0$ such that

$$
P(n, p, \psi(n, p)) \geq C_{\mathrm{EEP}} E(n, p, \psi(n, p))
$$

with

$$
C_{\mathrm{EEP}}^{-1}:=\frac{1}{2} e^{2\left(K_{\infty}+V_{\infty}\right)}(1+|\bar{C}|) \max \left\{\frac{\varepsilon}{\mu} e^{2\left(K_{\infty}+V_{\infty}\right)}(1+|\bar{C}|), \frac{1}{C_{\mathrm{F}}}\right\}\left(1+\frac{L(\Omega)}{\varepsilon} e^{2\left(K_{\infty}+V_{\infty}\right)}(1+|\bar{C}|)\right)
$$

where $C_{F}>0$ is defined in $(2)$ and $L(\Omega)>0$ is a constant satisfying Poincaré's inequality $\|\Psi\|_{L^{2}(\Omega)}^{2} \leq L(\Omega)\|\nabla \Psi\|_{L^{2}(\Omega)}^{2}$ for all $\Psi \in H^{1}(\Omega), \bar{\Psi}=0$.

Remark 1.4. In view of the above discussion, Theorem 1.3 can not hold as a functional inequality on a set of arbitrary states $(n, p, \psi)$ which includes the family $\left(N_{a, \psi}, P_{a, \psi}, \psi\right)$. In particular, it seems not admissible to include arbitrary potentials $\psi$ into the state space of the entropy-entropy production inequality without keeping in mind the solvability condition of the inhomogeneous Poisson equation. Therefore, maybe a better way of viewing Theorem 1.3 is - similar 
to the Keller-Segel model, see e.g. [Per07] - to formulate the entropy-entropy production inequality not on the state space of the parabolic-elliptic system (1), but for the corresponding non-local parabolic problem

$$
\begin{cases}\partial_{t} n=\nabla \cdot J_{n}(n, p)-R(n, p), & J_{n}(n, p):=\mu_{n}\left(\nabla n+n \nabla\left(G *(n-p-C)+V_{n}\right)\right), \\ \partial_{t} p=\nabla \cdot J_{p}(n, p)-R(n, p), & J_{p}(n, p):=\mu_{p}\left(\nabla p+p \nabla\left(-G *(n-p-C)+V_{p}\right)\right),\end{cases}
$$

where the self-consistent potential $\psi=\psi(n, p)$ is represented via the Green function $G$ (of second kind) of the inhomogeneous Poisson equation, i.e. $\psi=G *(n-p-C)$ such that $\bar{\psi}=0$.

Theorem 1.5 (Exponential Convergence to Equilibrium). Assume the existence of weak global non-negative solutions of (1)-(2) and (6) subject to non-negative initial data $\left(n_{I}, p_{I}\right)$ satisfying (8) (see e.g. [MRS90. Jün95. GGH96. GG96] and the references therein for typical definitions of weak solutions and [WMZ08] for results in this direction on $\mathbb{R}^{3}$ ) with sufficient regularity to satisfy the weak entropy production law

$$
E(n, p, \psi)\left(t_{1}\right)+\int_{t_{0}}^{t_{1}} P(n, p, \psi)(s) d s \leq E(n, p, \psi)\left(t_{0}\right), \quad \text { for all } \quad 0 \leq t_{0} \leq t_{1}<\infty .
$$

Then, these solutions decay exponentially to the equilibrium $\left(n_{\infty}, p_{\infty}, \psi_{\infty}\right)$ as a function of time $t \geq 0$ :

$$
E(n, p, \psi)(t) \leq E\left(n_{I}, p_{I}, \psi_{I}\right) e^{-K t}
$$

and

$$
\left\|n-n_{\infty}\right\|_{L^{1}(\Omega)}^{2}+\left\|p-p_{\infty}\right\|_{L^{1}(\Omega)}^{2}+\left\|\psi-\psi_{\infty}\right\|_{H^{1}(\Omega)}^{2} \leq\left(C+\frac{2}{\varepsilon}(1+L(\Omega))\right) E\left(n_{I}, p_{I}, \psi_{I}\right) e^{-K t}
$$

where $K:=C_{\mathrm{EEP}}$ and $C:=C_{\mathrm{CKP}}^{-1}$ are defined in Theorem 1.3 and Proposition 3.3 In addition, $\psi_{I} \in H^{1}(\Omega)$ is the unique solution of (9) with $f=n_{I}-p_{I}-C$ and $\overline{\psi_{I}}=0$.

\section{Derivation of an EEP-Inequality}

Proof of Proposition 1.2. A solution $\left(n_{\infty}, p_{\infty}, \psi_{\infty}\right)$ of 10 with $\overline{\psi_{\infty}}=0$ necessarily satisfies $J_{n}\left(n_{\infty}, \psi_{\infty}\right)=0$, $J_{p}\left(p_{\infty}, \psi_{\infty}\right)=0$ and $R\left(n_{\infty}, p_{\infty}\right)=0$, which implies $n_{\infty}=C_{n} e^{-\psi_{\infty}-V_{n}}$ and $p_{\infty}=C_{p} e^{\psi_{\infty}-V_{p}}$ for two constants $C_{n}, C_{p}>0$ with $C_{n} C_{p}=1$. By using arguments of e.g. [MRS90] and [WMZ08], there exists a unique equilibrium potential $\psi_{\infty} \in H^{1}(\Omega)$ with $\overline{\psi_{\infty}}=0$. The uniqueness of $C_{n}$ and $C_{p}$ (in terms of $\psi_{\infty}$ ) follows by monotonicity from $\overline{n_{\infty}}-\overline{p_{\infty}}=\bar{C}$. to

Having shown the existence, the continuity and boundedness of $\psi_{\infty}$ follow from applying [Trö09, Theorem 4.8]

$$
-\varepsilon \Delta \psi+\min \left\{C_{n} e^{-V_{n}}, C_{p} e^{-V_{p}}\right\} \psi+d(\cdot, \psi)=-C, \quad \hat{n} \cdot \nabla \psi=0 \quad \text { on } \partial \Omega,
$$

where $d(\cdot, \psi):=C_{p} e^{\psi-V_{p}}-C_{n} e^{-\psi-V_{n}}-\min \left\{C_{n} e^{-V_{n}}, C_{p} e^{-V_{p}}\right\} \psi$ constitutes a suitable monotone operator in terms of $\psi$. Note that [Trö09, Theorem 4.8] can not be used to directly prove the well-posedness of $\psi_{\infty}$ since the constants $C_{n}$ and $C_{p}$ depend non-locally on the solution.

Lemma 2.1. Under assumption (12), the following bounds on $C_{n}$ and $C_{p}$ hold in terms of $K_{\infty}, V_{\infty}$ and $|\bar{C}|$ :

$$
C_{n}, C_{p} \leq e^{K_{\infty}+V_{\infty}}(1+|\bar{C}|) \quad \text { and } \quad n_{\infty}, n_{\infty}^{-1}, p_{\infty}, p_{\infty}^{-1} \leq e^{2\left(K_{\infty}+V_{\infty}\right)}(1+|\bar{C}|) .
$$

Proof. We recall $C_{n} C_{p}=1$ and solve (11) for $C_{n}>0$ :

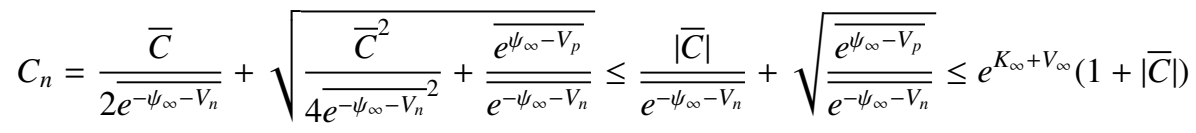

and the same bound holds for $C_{p}>0$ by an analog estimate. Finally, the bounds on $n_{\infty}, n_{\infty}^{-1}, p_{\infty}$ and $p_{\infty}^{-1}$ directly follow from the bounds on $C_{n}$ and $C_{p}$ and $C_{n} C_{p}=1$. 
Proposition 2.2. There exists an explicitly computable constant $c_{1}>0$ such that

$$
E(n, p, \psi) \leq c_{1} \int_{\Omega}\left(\frac{\left(n-n_{\infty}\right)^{2}}{n_{\infty}}+\frac{\left(p-p_{\infty}\right)^{2}}{p_{\infty}}\right) d x
$$

for all $n, p \in L^{2}(\Omega)$ where $\psi \in H^{1}(\Omega)$ is the unique solution of $(9)$ with $f=n-p-C$ and $\bar{\psi}=0$. More precisely, this inequality holds true for

$$
c_{1}:=1+\frac{L(\Omega)}{\varepsilon} e^{2\left(K_{\infty}+V_{\infty}\right)}(1+|\bar{C}|)
$$

Proof. From the elementary inequality $\ln x \leq x-1$ for $x>0$, we derive $n \ln \left(\frac{n}{n_{\infty}}\right)-\left(n-n_{\infty}\right) \leq n\left(\frac{n}{n_{\infty}}-1\right)-n+n_{\infty}=\frac{\left(n-n_{\infty}\right)^{2}}{n_{\infty}}$ and an analogous estimate for $p$ and $p_{\infty}$. Furthermore, integration by parts with homogeneous Neumann boundary conditions for $\psi$ and $\psi_{\infty}$ yields with $-\varepsilon \Delta\left(\psi-\psi_{\infty}\right)=\left(n-n_{\infty}\right)-\left(p-p_{\infty}\right)$

$$
\varepsilon \int_{\Omega}\left|\nabla\left(\psi-\psi_{\infty}\right)\right|^{2} d x=-\varepsilon \int_{\Omega}\left(\psi-\psi_{\infty}\right) \Delta\left(\psi-\psi_{\infty}\right) d x=\int_{\Omega}\left(\left(n-n_{\infty}\right)-\left(p-p_{\infty}\right)\right)\left(\psi-\psi_{\infty}\right) d x .
$$

Next, by applying Hölder's inequality and Young's inequality with some constant $\gamma>0$, we find

$$
\varepsilon \int_{\Omega}\left|\nabla\left(\psi-\psi_{\infty}\right)\right|^{2} d x \leq \frac{1}{2}\left(\frac{1}{\gamma}\left\|\left(n-n_{\infty}\right)-\left(p-p_{\infty}\right)\right\|^{2}+\gamma\left\|\psi-\psi_{\infty}\right\|^{2}\right) .
$$

Further, as a consequence of $\overline{\psi-\psi_{\infty}}=0$ and Poincaré's inequality, we estimate with $\gamma:=\varepsilon / L(\Omega)$

$$
\varepsilon \int_{\Omega}\left|\nabla\left(\psi-\psi_{\infty}\right)\right|^{2} d x \leq \frac{L(\Omega)}{\varepsilon}\left(\left\|n-n_{\infty}\right\|^{2}+\left\|p-p_{\infty}\right\|^{2}\right)+\frac{\varepsilon}{2}\left\|\nabla\left(\psi-\psi_{\infty}\right)\right\|^{2} .
$$

We thus arrive at

$\frac{\varepsilon}{2} \int_{\Omega}\left|\nabla\left(\psi-\psi_{\infty}\right)\right|^{2} d x \leq \frac{L(\Omega)}{\varepsilon} \int_{\Omega}\left(\left(n-n_{\infty}\right)^{2}+\left(p-p_{\infty}\right)^{2}\right) d x \leq \frac{L(\Omega)}{\varepsilon} e^{2\left(K_{\infty}+V_{\infty}\right)}(1+|\bar{C}|) \int_{\Omega}\left(\frac{\left(n-n_{\infty}\right)^{2}}{n_{\infty}}+\frac{\left(p-p_{\infty}\right)^{2}}{p_{\infty}}\right) d x$ where we have employed the bounds from Lemma 2.1. This proves the claim.

Proposition 2.3. There exists an explicitly computable constant $c_{2}>0$ such that

$$
\int_{\Omega}\left(\frac{\left(n-n_{\infty}\right)^{2}}{n_{\infty}}+\frac{\left(p-p_{\infty}\right)^{2}}{p_{\infty}}\right) d x \leq c_{2} P(n, p, \psi)
$$

for all $n, p \in L^{2}(\Omega)$ where $\psi \in H^{1}(\Omega)$ is the unique solution of 9 with $f=n-p-C$ and $\bar{\psi}=0$. In detail, this estimate is valid for

$$
c_{2}:=\frac{1}{2} e^{2\left(K_{\infty}+V_{\infty}\right)}(1+|\bar{C}|) \max \left\{\frac{\varepsilon}{\mu} e^{2\left(K_{\infty}+V_{\infty}\right)}(1+|\bar{C}|), \frac{1}{C_{\mathrm{F}}}\right\} .
$$

Proof. First, the entropy production (4) is bounded below by

$$
P(n, p, \psi) \geq c \int_{\Omega}\left(\frac{\varepsilon}{2}\left(\frac{n}{n_{\infty}}\left|\nabla \Phi_{n}\right|^{2}+\frac{p}{p_{\infty}}\left|\nabla \Phi_{p}\right|^{2}\right)+\frac{1}{2}\left(n p-e^{-V_{n}-V_{p}}\right) \ln \left(\frac{n p}{e^{-V_{n}-V_{p}}}\right)\right) d x
$$

where

$$
c:=\min \left\{\frac{2 \mu}{\varepsilon} e^{-2\left(K_{\infty}+V_{\infty}\right)} \frac{1}{1+|\bar{C}|}, 2 C_{\mathrm{F}}\right\} .
$$

A couple of elementary manipulations shows that

$$
\begin{aligned}
\frac{n}{n_{\infty}}\left|\nabla \Phi_{n}\right|^{2} & =\frac{n}{n_{\infty}}\left|\nabla\left(\psi+V_{n}+\ln n\right)\right|^{2}=\frac{n}{n_{\infty}}\left|\nabla\left(\psi+V_{n}+\ln C_{n}-\psi_{\infty}-V_{n}+\ln \left(\frac{n}{n_{\infty}}\right)\right)\right|^{2} \\
& =\frac{n}{n_{\infty}}\left|\nabla\left(\psi-\psi_{\infty}+\ln \left(\frac{n}{n_{\infty}}\right)\right)\right|^{2}=\frac{n}{n_{\infty}}\left|\nabla\left(\psi-\psi_{\infty}\right)\right|^{2}+2 \nabla\left(\psi-\psi_{\infty}\right) \cdot \nabla\left(\frac{n}{n_{\infty}}\right)+4\left|\nabla \sqrt{\frac{n}{n_{\infty}}}\right|^{2} \\
& \geq 2 \nabla\left(\psi-\psi_{\infty}\right) \cdot \nabla\left(\frac{n}{n_{\infty}}\right)=2 \nabla\left(\psi-\psi_{\infty}\right) \cdot \nabla\left(\frac{n}{n_{\infty}}-1\right) .
\end{aligned}
$$


Likewise, one finds $\frac{p}{p_{\infty}}\left|\nabla \Phi_{p}\right|^{2} \geq-2 \nabla\left(\psi-\psi_{\infty}\right) \cdot \nabla\left(\frac{p}{p_{\infty}}\right)=-2 \nabla\left(\psi-\psi_{\infty}\right) \cdot \nabla\left(\frac{p}{p_{\infty}}-1\right)$ and together

$$
\frac{\varepsilon}{2} \int_{\Omega}\left(\frac{n}{n_{\infty}}\left|\nabla \Phi_{n}\right|^{2}+\frac{p}{p_{\infty}}\left|\nabla \Phi_{p}\right|^{2}\right) d x \geq \varepsilon \int_{\Omega} \nabla\left(\psi-\psi_{\infty}\right) \cdot \nabla\left(\left(\frac{n}{n_{\infty}}-1\right)-\left(\frac{p}{p_{\infty}}-1\right)\right) d x .
$$

Hence, integration by parts yields

$$
\begin{aligned}
\frac{\varepsilon}{2} \int_{\Omega}\left(\frac{n}{n_{\infty}}\left|\nabla \Phi_{n}\right|^{2}+\frac{p}{p_{\infty}}\left|\nabla \Phi_{p}\right|^{2}\right) d x & \geq \int_{\Omega}\left(\left(n-n_{\infty}\right)-\left(p-p_{\infty}\right)\right)\left(\left(\frac{n}{n_{\infty}}-1\right)-\left(\frac{p}{p_{\infty}}-1\right)\right) d x \\
& =\int_{\Omega}\left(\frac{\left(n-n_{\infty}\right)^{2}}{n_{\infty}}+\frac{\left(p-p_{\infty}\right)^{2}}{p_{\infty}}\right) d x-\int_{\Omega}\left(\left(n-n_{\infty}\right)\left(p-p_{\infty}\right)\left(\frac{1}{n_{\infty}}+\frac{1}{p_{\infty}}\right)\right) d x
\end{aligned}
$$

and it remains to control the last term on the right hand side in order to complete the proof of Proposition 2.3 . Note that it is sufficient to consider this term on the set $\Omega^{+}:=\left\{x \in \Omega \mid\left(n(x)-n_{\infty}(x)\right)\left(p(x)-p_{\infty}(x)\right)>0\right\}$, where it is non-positive and can be estimated by the bounds on $n_{\infty}^{-1}$ and $p_{\infty}^{-1}$ from Lemma 2.1, i.e.

$$
\int_{\Omega}\left(\frac{\left(n-n_{\infty}\right)^{2}}{n_{\infty}}+\frac{\left(p-p_{\infty}\right)^{2}}{p_{\infty}}\right) d x \leq \frac{\varepsilon}{2} \int_{\Omega}\left(\frac{n}{n_{\infty}}\left|\nabla \Phi_{n}\right|^{2}+\frac{p}{p_{\infty}}\left|\nabla \Phi_{p}\right|^{2}\right) d x+2 e^{2\left(K_{\infty}+V_{\infty}\right)}(1+|\bar{C}|) \int_{\Omega^{+}}\left(n-n_{\infty}\right)\left(p-p_{\infty}\right) d x
$$

From $e^{-V_{n}-V_{p}}=n_{\infty} p_{\infty}$, the elementary inequality $(x-y) \ln \frac{x}{y} \geq 4(\sqrt{x}-\sqrt{y})^{2}$ for $x \geq 0, y>0$ and by following ideas of [GG96], we have

$$
\begin{aligned}
&\left(n p-e^{-V_{n}-V_{p}}\right) \ln \left(\frac{n p}{e^{-V_{n}-V_{p}}}\right)=\left(n p-n_{\infty} p_{\infty}\right) \ln \left(\frac{n p}{n_{\infty} p_{\infty}}\right) \geq 4\left(\sqrt{n p}-\sqrt{n_{\infty} p_{\infty}}\right)^{2} \\
&=\left(\left(\sqrt{n}-\sqrt{n_{\infty}}\right)\left(\sqrt{p}+\sqrt{p_{\infty}}\right)-\left(\sqrt{n}+\sqrt{n_{\infty}}\right)\left(\sqrt{p}-\sqrt{p_{\infty}}\right)\right)^{2}+4\left(\sqrt{n}-\sqrt{n_{\infty}}\right)\left(\sqrt{p}-\sqrt{p_{\infty}}\right)\left(\sqrt{n}+\sqrt{n_{\infty}}\right)\left(\sqrt{p}+\sqrt{p_{\infty}}\right) \\
& \geq 4\left(n-n_{\infty}\right)\left(p-p_{\infty}\right) .
\end{aligned}
$$

This shows that

$$
2 \int_{\Omega^{+}}\left(n-n_{\infty}\right)\left(p-p_{\infty}\right) d x \leq \frac{1}{2} \int_{\Omega^{+}}\left(n p-e^{-V_{n}-V_{p}}\right) \ln \left(\frac{n p}{e^{-V_{n}-V_{p}}}\right) d x
$$

which — together with (17) and (16) - yields

$$
\begin{aligned}
\int_{\Omega}\left(\frac{\left(n-n_{\infty}\right)^{2}}{n_{\infty}}+\frac{\left(p-p_{\infty}\right)^{2}}{p_{\infty}}\right) d x & \leq e^{2\left(K_{\infty}+V_{\infty}\right)}(1+|\bar{C}|) \int_{\Omega}\left(\frac{\varepsilon}{2}\left(\frac{n}{n_{\infty}}\left|\nabla \Phi_{n}\right|^{2}+\frac{p}{p_{\infty}}\left|\nabla \Phi_{p}\right|^{2}\right)+\frac{1}{2}\left(n p-e^{-V_{n}-V_{p}}\right) \ln \left(\frac{n p}{e^{-V_{n}-V_{p}}}\right)\right) d x \\
& \leq e^{2\left(K_{\infty}+V_{\infty}\right)}(1+|\bar{C}|) \max \left\{\frac{\varepsilon}{2 \mu} e^{2\left(K_{\infty}+V_{\infty}\right)}(1+|\bar{C}|), \frac{1}{2 C_{\mathrm{F}}}\right\} P(n, p, \psi)
\end{aligned}
$$

and, hence, the assertion.

Proof of Theorem 1.3. The proof is an immediate consequence of Proposition 2.2 and Proposition 2.3

\section{Convergence to the Equilibrium}

Lemma 3.1 (Classical Csiszár-Kullback-Pinsker inequality, see e.g. [DF08]). Let $f, g: \Omega \rightarrow \mathbb{R}$ be non-negative measureable functions. Then,

$$
\operatorname{RE}(f, g):=\int_{\Omega}\left(f \ln \left(\frac{f}{g}\right)-(f-g)\right) d x \geq \frac{3}{2 \bar{f}+4 \bar{g}}\|f-g\|_{L^{1}(\Omega)}^{2} .
$$

Lemma 3.2. Any entropy producing solution of (1) with initial relative entropy $E\left(n_{I}, p_{I}, \psi_{I}\right)$ satisfies

$$
\forall t \geq 0: \quad \bar{n}, \bar{p} \leq M_{1}, \quad \text { with } \quad M_{1}:=\frac{5}{2} e^{2\left(K_{\infty}+V_{\infty}\right)}(1+|\bar{C}|)+\frac{3}{4} E\left(n_{I}, p_{I}, \psi_{I}\right)
$$

where $\psi_{I} \in H^{1}(\Omega)$ is the unique solution of 9 with $f=n_{I}-p_{I}-C$ and $\overline{\psi_{I}}=0$. 
Proof. Lemma 3.1 and Young's inequality entail

$$
\bar{n} \leq \overline{n_{\infty}}+\left\|n-n_{\infty}\right\|_{L^{1}(\Omega)} \leq \overline{n_{\infty}}+\sqrt{\frac{2 \bar{n}+4 \overline{n_{\infty}}}{3}} \sqrt{\operatorname{RE}\left(n, n_{\infty}\right)} \leq \overline{n_{\infty}}+\frac{1}{3} \bar{n}+\frac{2}{3} \overline{n_{\infty}}+\frac{1}{2} \operatorname{RE}\left(n, n_{\infty}\right) .
$$

Solving this inequality for $\bar{n}$ yields $\bar{n} \leq \frac{5}{2} \overline{n_{\infty}}+\frac{3}{4} \operatorname{RE}\left(n, n_{\infty}\right)$. Therefore, we estimate with Lemma 2.1

$$
\bar{n} \leq \frac{5}{2} \overline{n_{\infty}}+\frac{3}{4} \operatorname{RE}\left(n, n_{\infty}\right) \leq \frac{5}{2} \overline{n_{\infty}}+\frac{3}{4} E(n, p, \psi) \leq \frac{5}{2} e^{2\left(K_{\infty}+V_{\infty}\right)}(1+|\bar{C}|)+\frac{3}{4} E\left(n_{I}, p_{I}, \psi_{I}\right)
$$

due to the monotonicity of the entropy functional. In the same way follows the bound on $\bar{p}$.

Proposition 3.3. For all $n, p \in L^{2}(\Omega)$ satisfying $\bar{n}, \bar{p} \leq M_{1}$ and $\psi \in H^{1}(\Omega)$ being the corresponding unique solution of (9) with $f=n-p-C$ and $\bar{\psi}=0$, the following Csiszár-Kullback-Pinsker type inequality holds:

$$
E(n, p, \psi) \geq C_{\mathrm{CKP}}\left(\left\|n-n_{\infty}\right\|_{L^{1}(\Omega)}^{2}+\left\|p-p_{\infty}\right\|_{L^{1}(\Omega)}^{2}\right), \quad \text { with } \quad C_{\mathrm{CKP}}:=\left(3 e^{2\left(K_{\infty}+V_{\infty}\right)}(1+|\bar{C}|)+\frac{1}{2} E\left(n_{I}, p_{I}, \psi_{I}\right)\right)^{-1} .
$$

where $E\left(n_{I}, p_{I}, \psi_{I}\right)$ is the initial relative entropy and $\psi_{I} \in H^{1}(\Omega)$ is the unique solution of (9) with $f=n_{I}-p_{I}-C$ and $\overline{\psi_{I}}=0$.

Proof. We observe that

$$
E(n, p, \psi) \geq \int_{\Omega}\left(n \ln \frac{n}{n_{\infty}}-\left(n-n_{\infty}\right)+p \ln \frac{p}{p_{\infty}}-\left(p-p_{\infty}\right)\right) d x \geq \frac{3}{2 \bar{n}+4 \overline{n_{\infty}}}\left\|n-n_{\infty}\right\|_{L^{1}(\Omega)}^{2}+\frac{3}{2 \bar{p}+4 \overline{p_{\infty}}}\left\|p-p_{\infty}\right\|_{L^{1}(\Omega)}^{2}
$$

thanks to Lemma 3.1. The proof then follows from the assumption $\bar{n}, \bar{p} \leq M_{1}$ and Lemma 2.1 .

$$
E(n, p, \psi) \geq 3\left(9 e^{2\left(K_{\infty}+V_{\infty}\right)}(1+|\bar{C}|)+\frac{3}{2} E\left(n_{I}, p_{I}, \psi_{I}\right)\right)^{-1}\left(\left\|n-n_{\infty}\right\|_{L^{1}(\Omega)}^{2}+\left\|p-p_{\infty}\right\|_{L^{1}(\Omega)}^{2}\right) .
$$

Proof of Theorem 1.5 . The statement of Theorem 1.5 follows directly from a Gronwall argument together with Theorem 1.3 and Proposition 3.3 .

Acknowledgements. The second author has been supported by the International Research Training Group IGDK 1754 "Optimization and Numerical Analysis for Partial Differential Equations with Nonsmooth Structures", funded by the German Research Council (DFG) and the Austrian Science Fund (FWF): [W 1244-N18].

\section{References}

[DF06] L. Desvillettes, K. Fellner, Exponential decay toward equilibrium via entropy methods for reaction-diffusion equations, J. Math. Anal. Appl. 319 (2006), 157-176.

[DF08] L. Desvillettes, K. Fellner, Entropy methods for reaction-diffusion equations: slowly growing a-priori bounds, Revista Matematica Iberoamericana 24 (2008), 407-431.

[DF14] L. Desvillettes, K. Fellner, Exponential Convergence to Equilibrium for a Nonlinear Reaction-Diffusion Systems Arising in Reversible Chemistry, System Modelling and Optimization, IFIP AICT 443 (2014), 96-104.

[DFT17] L. Desvillettes, K. Fellner, B. Q. Tang, Trend to equilibrium for reaction-diffusion systems arising from complex balanced chemical reaction networks, SIAM Journal on Math. Analysis 49 no.4 (2017), 2666-2709.

[DFM08] M. Di Francesco, K. Fellner, P. Markowich, The entropy dissipation method for inhomogeneous reaction-diffusion systems, Proc. Royal Soc. A 464 (2008), 3272-3300.

[FT17] K. Fellner, B.Q. Tang, Explicit exponential convergence to equilibrium for mass action reaction-diffusion systems with detailed balance condition, Nonlinear Analysis 159 (2017), 145-180.

[GG96] H. Gajewski, K. Gärtner, On the Discretization of van Roosbroeck's Equations with Magnetic Field, Z. angew. Math. Mech. 76 (1996), $247-264$.

[GZ10] I. Gentil, B. Zegarlinski, Asymptotic behaviour of a general reversible chemical reaction-diffusion equation, Kinet. Relat. Models 3 (2010), $427-444$ 
[GGH96] A. Glitzky, K. Gröger, R. Hünlich, Free energy and dissipation rate for reaction-diffusion processes of electrically charged species, Appl. Anal. 60 (1996), 201-217.

[GH97] A. Glitzky, R. Hünlich, Energetic estimates and asymptotics for electro-reaction-diffusion systems, Z. Angew. Math. Mech. 77 (1997), $823-832$.

[Grö83] K. Gröger, Asymptotic Behavior of Solutions to a Class of Diffusion-Reaction Equations, Math. Nachr. 112 (1983), 19-33.

[Grö92] K. Gröger, Free energy estimates and asymptotic behaviour of reaction-diffusion processes, Preprint 20, Institut für Angewandte Analysis und Stochastik, Berlin, 1992.

[HHMM] J. Haskovec, S. Hittmeir, P. A. Markowich, A. Mielke, Decay to equilibrium for energy-reaction-diffusion systems, arXiv:1602.05696.

[Jün95] A. Jüngel, Qualitative behavior of solutions of a degenerate nonlinear drift-diffusion model for semiconductors, Math. Models Methods Appl. Sci. 5 (1995), 497-518.

[MHM15] A. Mielke, J. Haskovec, P. A. Markowich, On uniform decay of the entropy for reaction-diffusion systems, J. Dyn. Diff. Eqs. 27 (2015), 897-928.

[MRS90] P.A.Markowich, C. Ringhofer, C. Schmeiser, Semiconductor Equations, Springer (1990).

[Per07] B. Perthame, Transport equations in biology, Birkhäuser (2007).

[Trö09] F. Tröltzsch, Optimale Steuerung partieller Differentialgleichungen, Vieweg+Teubner (2009).

[WMZ08] H. Wu, P. A. Markowich, S. Zheng, Global existence and asymptotic behavior for a semiconductor drift-diffusion-Poisson model, Math. Models Methods Appl. Sci. 18 (2008), 443-487. 